\title{
Early diagnosis and precise treatment of hepatocellular carcinoma
}

\author{
Wenxing Zhao $^{1^{*}}$ \\ ${ }^{1}$ Northwest A\&F University, Shaanxi 712100, China
}

\begin{abstract}
Liver cancer is called the "king of cancer" because of its extremely high mortality rate and lack of effective treatment methods. Effective early diagnosis of liver cancer and advance discovery period of liver cancer can effectively improve the survival rate and life cycle. Early diagnosis can be done by traditional US, CT, AFP level measurement and other methods, or by biological markers such as GPC-3, micro-RNA, and detection of some specific cell signal factors. Precision treatment is often carried out with a personalized treatment plan for the patient and a targeted therapy at the molecular level.
\end{abstract}

\section{Introduction}

Liver cancer refers to hepatocellular carcinoma (hepatocellular carcinoma, HCC), which is the second most fatal cancer and also the sixth most common type in the world with an increasing incidence [1]. The survival rate of HCC patients is very low. In China, the 5-year survival rate of HCC patients is only $12.1 \%$, which is the second lowest survival rate among all types of cancer [2]. In order to solve this problem, the early diagnosis of hepatocellular carcinoma is very important. According to certain symptoms of the patient, the corresponding precise treatment plan is formulated, which is of great significance for the choice of treatment method and increase the survival rate of the patient. This article gives a brief introduction to liver cancer, collects research results and progress in recent years, analyzes the current common early diagnosis technical methods and some precision treatment methods for hepatocellular liver cancer, and summarizes the clinical and scientific theories, which provides some ideas for the improvement of clinical and scientific theories.

\section{Liver cancer}

\subsection{Introduction of liver cancer}

Primary liver cancer has the second highest mortality rate of cancer-related malignant tumors in the world, and the second highest rate of tumors in China [3]. More than $90 \%$ of primary liver cancers are hepatocellular carcinoma (HCC), and about $5 \%-10 \%$ of primary liver cancers are cholangiocarcinoma (cholangiocarcinoma).

\subsubsection{Causes of disease}

The main potential risk of liver cancer is liver cirrhosis, which usually results from chronic viral infection (hepatitis B or C), or alcoholic liver disease. For most patients, $\mathrm{HCC}$ is related to chronic liver damage caused by hepatitis virus infection, alcohol abuse, or non-alcoholic liver steatosis, which not only complicates the choice of the treatment, but also worsens the tumor, thus affecting the chance of survival of patients [4].

\subsubsection{High-risk groups}

There are high-risk groups of hepatocellular carcinoma. Among high-risk groups, in addition to the originally susceptible groups, some of them are caused by the influence of special environments.

- People infected with hepatitis B or C viruses.This group is the main high-risk group of liver cancer in China, which accounts for a large proportion of liver cancer patients. Viral infection causes hepatitis, and hepatitis leads to liver cirrhosis, which in turn liver cancer occurs. A wide range of popular antibody vaccination can effectively reduce the proportion of infections.

- People with family history. The occurrence of liver cancer and liver disease has a certain genetic tendency, and the hepatitis virus is highly infectious. This part of the population should timely do check-ups and replant vaccines to prevent problems before they occur.

- People with other diseases. Some people suffering from autoimmune diseases, diabetes, and other diseases may cause complications in the liver due to low immunity and long-term damage. 


\subsection{Special environments lead to High-risk situations.}

- Excessive alcoholics. Long-term excessive drinking may cause oxidation or necrosis of the liver, leading to alcoholic liver disease, liver cirrhosis and liver cancer.

- Unclean environment. Drinking heavily polluted water, and if it contains too many heavy metals and contains various toxins, then that may cause liver disease.

- Parasite infection. Studies have shown that schistosomiasis infection can cause liver damage and increase the risk of liver cancer.

- Contact with toxic substances. Ingestion of food contaminated by Aspergillus flavus, exposure to some carcinogenic substances, etc. will increase the chance of liver cancer.

\section{Early diagnosis}

\subsection{Significance of early diagnosis}

For early HCC patients, various methods such as surgery, tumor ablation, or liver transplantation can be used for treatment. Patients receiving treatment can often have a higher survival rate and a longer survival period. However, in China, most of the diagnosed HCC patients are in the late or advanced stage of cancer. When surgical intervention is not possible, as the tumor develops, the liver function will decline, and intrahepatic and extrahepatic metastasis will occur (mainly to Lungs and bones) [5], coupled with the high degree of malignancy and rapid development of advanced liver cancer, the therapeutic effects of chemotherapy drugs and other treatments are not ideal, which seriously affects the survival status of HCC patients and greatly reduces the survival rate.

Therefore, the early diagnosis of liver cancer is extremely important. However, it is precise because of the difficulty of early diagnosis that this situation occurred. Therefore, searching for reasonable early diagnosis methods and summarizing and reflecting on the existing methods have become the top priority.

\subsection{Methods of early diagnosis}

\subsubsection{Traditional diagnostic methods}

- Ultrasonic imaging technology (US). The most widely available method of screening is ultrasound, which does not involve ionizing radiation. In patients with liver cirrhosis, the sensitivity of the test is between $60 \%-80 \%$, and the specificity can be greater than $90 \%$. This method is the first choice for HCC screening in patients with liver cirrhosis and should be performed every 6 months [6].

- Computerized tomography (CT)/MRI. The definite diagnosis of HCC requires abdominal enhanced CT or magnetic resonance scan (MRI). The sensitivity of abdominal enhanced $\mathrm{CT}$ is $68 \%$, and the specificity is
$93 \%$ [7]. The sensitivity of MRI scan is $85 \%$, and the specificity is $93 \%$ [7]. In the arterial phase, enhanced CT and MRI scans can often show that nodules with important enhanced signals become highly sensitive or excessively attenuated. In the portal phase and late stage, HCC usually shows rapid disappearance of angiography, low density or high attenuation compared with other liver parenchyma.

- Measurement of serum alpha-fetoprotein (AFP) level. Since the 1980s, the determination of serum APF concentration has been one of the routine methods for HCC diagnosis. Studies have shown that the best cut-off range of AFP is $16-20 \mathrm{ng} / \mathrm{ml}$, and the specificity and sensitivity are $90 \%$ and $60 \%$, respectively [8]. But for early diagnosis results, its sensitivity is very low. There is no significant change in the concentration of AFP in a large part of small HCC cases. Only when the diameter of the tumor is $>3 \mathrm{~cm}$, the sensitivity increases from $25 \%$ to $52 \%[9]$. Therefore, for early diagnosis, this method is not particularly effective and can only be used as a reference for other detection methods.

\subsubsection{Biological methods}

With the rapid development of biology, methods for early diagnosis of hepatocellular carcinoma at the molecular level have also been developed through biomarkers and specific genetic changes.

- Glypican-3 (GPC-3). Glypican-3 (Glypican-3, GPC-3) has high sensitivity and specificity in the serum and case specimens of patients with hepatocellular carcinoma, and is different from cholangiocellular carcinoma and other liver diseases. It is specifically and highly expressed in the serum and pathological specimens of patients with hepatocellular carcinoma. It is a specific marker for hepatocellular carcinoma. It also reminds us of potential targets for cancer treatment. In addition, the expression level of GPC-3 combined with the detection result of AFP can effectively increase the detection rate of HCC.

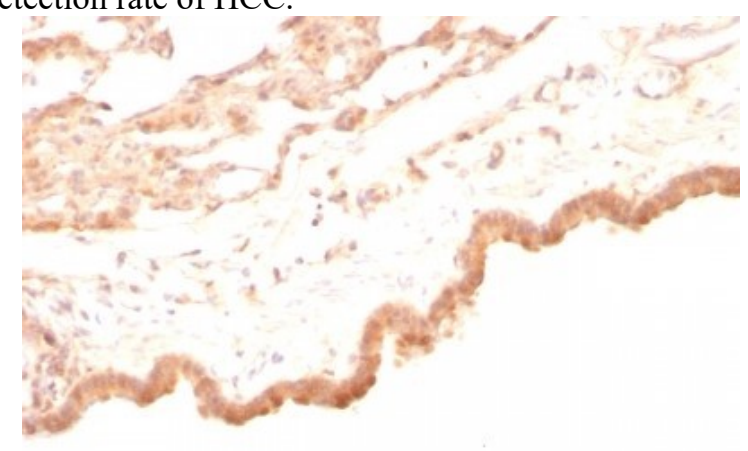

Fig.1 Glypican 3 Antibody

- Micro RNA. Studies have shown that many miRNAs will change after the occurrence of HCC, such as mi-12, mi-122, mi-10b, mi-206, mi-192-5p, mi-221, etc., may have the potential value of early diagnosis of $\mathrm{HCC}$. And some may become key target genes for treatment. 


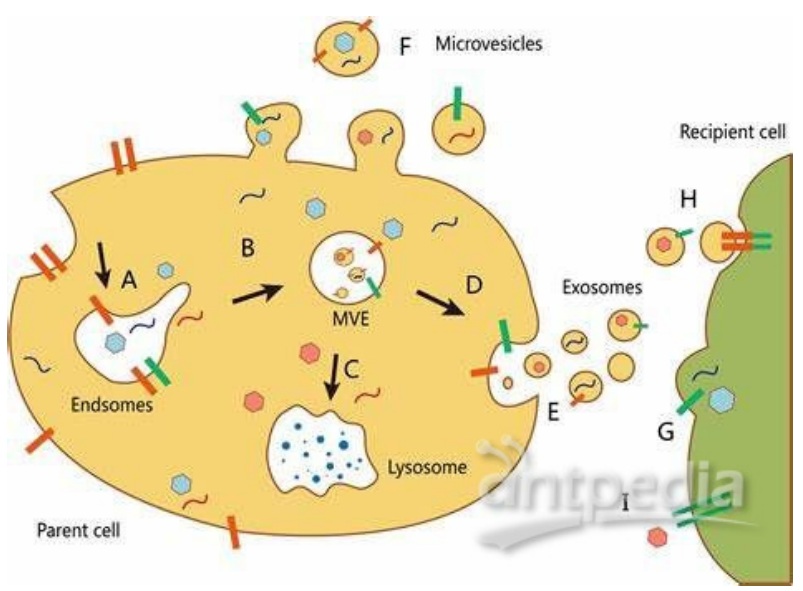

Fig. 2. RNA sequencing

\subsubsection{Other methods}

Mutations in genes related to the p53 signaling pathway that can cause chromosomal abnormalities, such as $\mathrm{CKS1}$, p53, and $\beta$-catenin signaling pathway related genes, may cause the occurrence of HCC. In addition, studies have also shown that some long-chain non-coding RNAs, such as H19, MEG3, MALAT1, etc. have a great relationship with the occurrence of $\mathrm{HCC}[10-12]$.

\section{Precision treatment}

\subsection{Differences from traditional treatments}

Precision therapy is a new medical concept and medical model developed with the rapid advancement of genome sequencing technology and the cross application of bioinformatics and big data science based on personalized medicine. Its essence is to use genomics, proteomics and other cutting-edge technologies in medicine to analyze and identify biomarkers for a large sample of people and specific disease types, so as to accurately find the cause and target, and achieve personalized accuracy for specific patients The purpose of treatment can greatly improve the effectiveness of disease diagnosis and treatment and defense.

\subsection{Methods of precision treatment}

\subsubsection{Clinical level}

Precision treatment at the clinical level is basically based on clear data such as the clinical stage of the tumor, pathological type, and whether there are tumor thrombi in the vessel as prognostic indicators, and refer to relevant materials and documents to formulate an individualized treatment plan. Specific methods include hepatectomy, catheter therapy, puncture therapy, systemic or systemic therapy (sorafenib, levatinib), immunotherapy (sorafenib), chemotherapy, radiotherapy, etc.

\subsubsection{Molecular level}

Molecular-level therapy is mainly carried out through molecular targeted drugs. Verteporfin can selectively inhibit YAP-induced tumorigenesis, and experiments have shown that it can inhibit the carcinogenic activity of YAP without affecting the size and safety of animal livers. Multi-targeting tyrosine kinase inhibitors sunitinib, erlotinib, epidermal growth factor receptor inhibitor gefitinib, and anti-vascular endothelial growth factor humanized monoclonal antibody bevacizumab can also be targeted for HCC treatment drug.

\section{Conclusion}

The degree of malignancy and mortality of hepatocellular carcinoma is extremely high. Cirrhosis of the liver is the main cause of hepatocellular carcinoma, which is often caused by a chronic viral infection. There are high-risk and susceptible people in the onset of HCC. These people are more likely to cause $\mathrm{HCC}$ due to viral infections, environmental damage, poor diet, and other reasons. Early diagnosis can effectively control the progression of hepatocellular carcinoma. Traditional methods include ultrasound imaging technology (US), tomography (CT), AFP level measurement, etc. Modern methods can also be detected by biological molecular markers, such as GPC-3. Some microRNAs, or cytokines involved in related signaling pathways. Precise treatment for patients with hepatocellular carcinoma. At the clinical level, individualized treatment plans are formulated according to the condition and the characteristics of the patients. At the molecular level, targeted treatment through targeted drugs is effective in reducing $\mathrm{HCC}$ mortality and increasing survival. It has a very important meaning.

In the past 10 years, the treatment of $\mathrm{HCC}$ has made great progress. However, early diagnosis of liver cancer patients can effectively improve the survival rate of patients. Patients with $\mathrm{HCC}$ at an early stage can also have more treatment options. For patients with different disease periods, the use of personalized precision treatment can also effectively improve the level of disease treatment. However, we still have many problems in these two areas that we look forward to exploring and solving. We still have a long way to go on the road to liver cancer treatment.

\section{Acknowledgement}

First of all I want to thank my mentor. It was his careful guidance that opened my horizons, enriched my knowledge, and made me use it for a lifetime. Especially the teacher's rigorous and serious teaching style impressed me deeply. In my future study, life and work, I will definitely uphold the teacher's style and be a rigorous and serious person.

I want to thank my classmates. When I was lonely and helpless, they were the ones who inspired me to move forward; when I was confused, they were the ones who guided me forward. 


\section{References}

1. R. Salem, RJ. Lewandowski, Chemoembolization and radioembolization for hepatocellular carcinoma. Clin Gastroenterol Hepatol. 2013;11:604

2. Zeng H, Chen W, Zheng R, Zhang S, Ji JS, Zou X, et al. Changing cancer survival in China during 2003-15: a pooled analysis of 17 population-based cancer registries. Lancet Glob Health. 2018;6(5): e555-67.

3. Zhou M, Wang $\mathrm{H}$, Zeng $\mathrm{X}$, et al. Mortality, morbidity, and risk factors in China and its provinces, 1990-2017: a systematic analysis for the Global Burden of Disease Study 2017 [published correction appears in Lancet. 2020 Jul 4;396 (10243):26]. Lancet. 2019; 394(10204):1145-1158. doi:10.1016/S0140-6736(19)30427-1

4. Medavaram S, Zhang Y. Emerging therapies in advanced hepatocellular carcinoma. Exp Hematol Oncol. 2018;7:17.

5. Kumar V, Abbas AK, Aster JC, editors. Robbins \& Contran. Pathologic Basis of Diseases. 9th ed. Philadelphia: Elsevier; 2014.

6. Clavien PA, Lesurtel M, Bossuyt PM, et al. Recommendations for liver transplantation for hepatocellular carcinoma: an international consensus conference report. Lancet Oncol. 2012;13:e11-22.

7. Colli A, Fraquelli M, Casazza G, Massironi S, Colucci A, Conte D, Duca P. Accuracy of ultrasonography, spiral CT, magnetic resonance, and alpha fetoprotein in diagnosing hepatocellular carcinoma: a systematic review. Am J Gastroenterol. 2006;101:513-23.

8. Li D, Mallory T, Satomura S . AFP-L3: a new generation of tumor marker for hepatocellular carcinoma.[J]. Clinica Chimica Acta, 2001, 313(1-2):15-19.

9. Raphaël Saffroy, Pham P, Maâmar Reffas, et al. New perspectives and strategy research biomarkers for hepatocellular carcinoma[J]. Clinical Chemistry \& Laboratory Medicine, 2007, 45(9):1169-1179.

10. Matouk I J , Degroot N, Mezan S, et al. The H19 Non-Coding RNA Is Essential for Human Tumor Growth[J]. Plos One, 2012, 2.

11. Braconi, Kogure, Valeri, et al. microRNA-29 can regulate expression of the long non-coding RNA gene MEG3 in hepatocellular cancer.[J]. Oncogene, 2011.

12. Ming-chun, Lai, Zhe, et al. Long non-coding RNA MALAT-1 overexpression predicts tumor recurrence of hepatocellular carcinoma after liver transplantation[J]. Medical Oncology, 2012. 